Rosemary E. Gale, Katarina Lamb, Christopher Allen, Dima El-Sharkawi, Cassandra Stowe, Sarah Jenkinson, Steven Tinsley, Glenda Dickson, and David C. Linch, University College London Cancer Institute, London; and Alan K. Burnett and Robert K. Hills, Cardiff University School of Medicine, Cardiff, United Kingdom.

Published online ahead of print at www.jco.org on May 11, 2015.

Supported by Leukaemia and Lymphoma Research, UK and the United Kingdom Medical Research Council. The work was done at University College London Hospitals/University College London, which received a proportion of funding from the United Kingdom Department of Health's National Institute for Health Research Biomedical Research Centres funding scheme.

Presented at the 56th Annual Meeting of the American Society of Hematology, San Francisco, CA, December 6-9 2014

Authors' disclosures of potential conflicts of interest are found in the article online at www.jco.org. Author contributions are found at the end of this article.

Corresponding author: Rosemary E. Gale, PhD, Department of Haematology, University College London Cancer Institute, Paul O'Gorman BIdg, 72 Huntley St, London, WC1E 6DD United Kingdom; e-mail: rosemary.gale@ucl.ac. uk.

C 2015 by American Society of Clinica Oncology

$0732-183 X / 15 / 3318 w-2072 w / \$ 20.00$

DOI: 10.1200/JCO.2014.59.2022

\title{
Simpson's Paradox and the Impact of Different DNMT3A Mutations on Outcome in Younger Adults With Acute Myeloid Leukemia
}

Rosemary E. Gale, Katarina Lamb, Christopher Allen, Dima El-Sharkawi, Cassandra Stowe, Sarah Jenkinson, Steven Tinsley, Glenda Dickson, Alan K. Burnett, Robert K. Hills, and David C. Linch

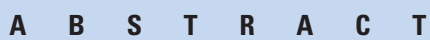

\section{Purpose}

To evaluate the impact of DNMT3A mutations on outcome in younger patients with cytogenetic intermediate-risk acute myeloid leukemia.

\section{Patients and Methods}

Diagnostic samples from 914 patients ( $97 \%<60$ years old) were screened for mutations in DNMT3A exons 13 to 23. Clinical outcome was evaluated according to presence or absence of a mutation and stratified according to type of mutation (R882, non-R882 missense, or truncation).

\section{Results}

DNMT3A mutations (DNMT3AMUT) were identified in 272 patients (30\%) and associated with a poorer prognosis than wild-type DNMT3A, but the difference was only seen when the results were stratified according to NPM1 genotype. This example of Simpson's paradox results from the high coincidence of DNMT3A and NPM1 mutations $180 \%$ of patients with DNMT3A $A^{\mathrm{MUT}}$ had NPM1 mutations), where the two mutations have opposing prognostic impact. In the stratified analyses, relapse in patients with DNMT3A $A^{\text {MUT }}$ was higher (hazard ratio, $1.35 ; 95 \% \mathrm{Cl}, 1.07$ to $1.72 ; P=$ .01 ), and overall survival was lower (hazard ratio, 1.37; $95 \% \mathrm{Cl}, 1.12$ to $1.87 ; P=.002$ ). The impact of DNMT3A $A^{\text {MUT }}$ did not differ according to NPM1 genotype (test for heterogeneity: relapse, $P=$ .4; overall survival, $P=.9$ ). Further analysis according to the type of DNMT3A mutation indicated that outcome was comparable in patients with R882 and non-R882 missense mutants, whereas in those with truncation mutants, it was comparable to wild-type DNMT3A.

\section{Conclusion}

These data confirm that presence of a DNMT3A mutation should be considered as a poor-risk prognostic factor, irrespective of the NPM1 genotype, and suggest that further consideration should be given to the type of DNMT3A mutation.

\section{J Clin Oncol 33:2072-2083. (C) 2015 by American Society of Clinical Oncology}

\section{INTRODUCTION}

The development of high-throughput technologies has had a major influence on uncovering the molecular landscape of tumors and, in acute myeloid leukemia (AML), has revealed a hitherto unrecognized degree of complexity and heterogeneity that presents considerable challenges for the stratification of risk-directed therapy. Whole-genome/exome sequence data from 200 patients with AML showed that the incidence of the majority of recurrently mutated genes detected was less than $10 \%$ of all patients, but FLT3 internal tandem duplications (FLT3 ${ }^{\mathrm{ITD}}$ ) and mutations in the NPM1 $\left(N P M 1^{\mathrm{MUT}}\right)$ and DNA methyltransferase $3 \mathrm{~A}\left(D N M T 3 A^{\mathrm{MUT}}\right)$ genes were much more frequent, each occurring in more than $25 \%$ of patients. ${ }^{1}$ All three mutations predominate in patients with cytogenetically intermediate-risk (IR) AML, and they are often coincident. ${ }^{2-9}$ FLT3 and NPM1 are now routinely screened at diagnosis, with general consensus that FLT3 $3^{\mathrm{ITD}}$ is associated with an adverse impact and NPM1 ${ }^{\mathrm{MUT}}$ with a favorable impact on prognosis, ${ }^{10,11}$ and patients with wild-type (WT) FLT3 and NPM1 ${ }^{\mathrm{MUT}}$ are not recommended for stem-cell transplantation in first remission. ${ }^{12,13}$ The role of $D N M T 3 A^{\mathrm{MUT}}$ in prognostication is less clear. Although most studies have reported that mutations are associated with worse overall survival (OS), reviewed in recent metaanalyses, ${ }^{14,15}$ the largest study to date that included 1,060 younger adult patients with IR cytogenetics found that DNMT3 $A^{\mathrm{MUT}}$ had no significant impact on survival end points, either overall or in the normal karyotype (NK) subgroup. ${ }^{9}$ Furthermore, in 
studies where an adverse impact has been recorded, there are variable results for the different FLT3/NPM1 genotypic subgroups. ${ }^{3-7,9}$ Of particular importance to patient management, there is controversy on the impact of the mutations in low-risk patients with IR/NK cytogenetics and FLT3 ${ }^{\mathrm{WT}} N P M 1^{\mathrm{MUT}}$ genotype, with studies reporting either no impact ${ }^{4,7,9}$ or adverse impact. ${ }^{5,6}$

DNMT3A is a DNA methyltransferase that is responsible for de novo methylation of $\mathrm{CpG}$ dinucleotides. It has three conserved functional domains, the C-terminal methyltransferase catalytic domain encoded by exons 16 to 23, an N-terminal PWWP domain (exons 8 and 9) that binds to specific lysine residues in methylated histones, and an ADD zinc finger domain (exons 13 to 15) that interacts with nonmethylated H3K4. ${ }^{16}$ Mutations were first identified in AML from array-based or whole-genome/exome sequencing of patient samples. ${ }^{2,17,18}$ Missense mutations at residue R882 are particularly frequent, occurring in approximately two thirds of mutated cases. ${ }^{2-9,18}$ Other mutations are found throughout the gene, predominantly in exons 13 to 23. They include missense, nonsense, frameshift, and splice site mutations and are usually heterozygous.

There is limited information on the impact of the different mutations, with reports of either no difference between R882 and nonR882 mutations $s^{2,4,6,7}$ or variable impact in selected groups ${ }^{8,9}$ and no data comparing the impact of missense and truncating mutations, although they may have differing functional consequences. The major R882H mutation disrupts the DNMT3A homodimer interface and acts in a dominant-negative manner, leading to reduced homotetramer formation and much lower catalytic activity. ${ }^{19-21}$ Other missense mutations also occur at the dimer interface, but some are located at the tetramer interface and may have more modest effects on catalytic activity but reduce overall methylation. ${ }^{22}$ Truncating mutations, however, are more likely to lead to nonsense-mediated decay ${ }^{23}$ and haploinsufficiency than dominant-negative activity. In mouse models, heterozygous Dnmt3a deletion had no apparent effect on phenotype, ${ }^{24}$ and primary transplant recipients of Dnmt3a-null hematopoietic stem cells did not have evident changes in hematopoiesis. ${ }^{25,26}$ Furthermore, high-resolution methylation analysis has now shown focal hypomethylation at specific CpG residues in R882mutated patients that is not seen in the non-R882-mutated patients, with a distinct hierarchical clustering of the R882-mutated patients away from the non-R882-mutated patients. ${ }^{21}$

Because knowledge of DNMT3A mutations and their impact on clinical outcome may influence both gene screening strategies and therapy risk stratification, we have screened samples from a large cohort of 914 younger adult IR patients in whom there is extensive follow-up data and evaluated outcome according to the type of DNMT3A mutation detected.

\section{PATIENTS AND METHODS}

\section{Patient Cohort}

Genomic DNA was available from diagnostic samples of 914 patients with IR cytogenetics, as defined by the Medical Research Council classification, ${ }^{27}$ uniformly treated on the United Kingdom Medical Research Council AML10 and AML12 trials between 1988 and 2002; 54\% of samples were from bone marrow, $44 \%$ were from peripheral blood, and for $2 \%$, the source was not known. Ethical approval for the trials and tissue collection for research was obtained from the Multi-Centre Research Committee of Wales. Informed consent was obtained in accordance with the Declaration of Helsinki. Patient characteristics are listed in Table 1. Median age at presentation was 43 years (range, 15 to 68 years); only 24 patients ( $3 \%$ ) were $\geq 60$ years old. All samples had known $F L T 3^{\mathrm{ITD}}$ and FLT3 tyrosine kinase domain, NPM1, CCAAT/ enhancer binding protein- $\alpha$ (CEBPA), and isocitrate dehydrogenase (IDH) 1 and IDH2 genotype. ${ }^{28-32}$ Compared with the 1,996 IR patients treated on these trials who were not included in the study, the investigated patients had significantly higher WBC counts $(P<.001)$ but no differences in median age, sex, incidence of secondary disease, or proportion who received a stem-cell transplantation in first remission (Appendix Table A1, online only). The investigated cohort, compared with patients not included in the study, had a borderline higher remission rate $(87 \% v 84 \%$, respectively; $P=.03)$ and better 5 -year OS (39\% $v 34 \%$, respectively; $P=.02$ ), but there was no significant difference in cumulative incidence of relapse (CIR; $49 \% v 53 \%$, respectively; $P=.1$ ).

\section{DNMT3A Screening and Mutant Quantification}

Amplicons of DNMT3A exons 13 to 23 were screened by denaturing high-performance liquid chromatography (Appendix and Appendix Table A2, online only). The common R882H and R882C mutations were confirmed by restriction enzyme digestion; other samples with abnormal chromatograms were sequenced. The relative mutant level for samples with R882H and R882C mutations was quantified using pyrosequencing and expressed as a relative proportion of total DNMT3A alleles.

\section{Statistical Methods and Clinical End Points}

Details of the trial protocols have been previously published. ${ }^{33,34}$ The AML12 trial is registered at http://www.controlled-trials.com under ISRCTN17833622. Of the 914 patients, 100 underwent allogeneic transplantation from a sibling donor and 17 underwent transplantation from a matched unrelated donor in first remission.

Clinical end points are defined in the Appendix. Mantel-Haenszel and $\chi^{2}$ tests were used to test for differences in demographic and clinical data by DNMT3A status. Kaplan-Meier curves were constructed for survival data and compared by means of the log-rank test, with standard tests for heterogeneity between subgroups. ${ }^{35}$ Surviving patients were censored on August 9, 2010, with follow-up complete for $98 \%$ of patients. Median follow-up was 13.4 years (range, 5.2 to 21.9 years). Multivariable logistic regression analysis was used to find the factors most closely associated with complete remission, and multivariable Cox analysis was used for CIR and OS. Results were adjusted for age, WHO performance status, $\log (\mathrm{WBC})$, secondary disease, $F L T 3^{\mathrm{ITD}}$, and NPM1 genotype. Odds ratios or hazard ratios (HRs) and 95\% CIs are quoted for end points. In all cases, a ratio of less than 1 indicates benefit for a mutation. All $P$ values are two-tailed.

\section{RESULTS}

\section{DNMT3A Mutation Analysis}

Overall, 278 mutations were detected in 272 (30\%) of 914 patients (Fig 1A and Appendix Table A3, online only); 175 (63\%) were missense R882 mutations. The remaining mutations were distributed throughout the gene and included 63 missense mutations, 37 mutations classified as truncations (12 nonsense, 13 frameshift, 12 at splice sites), and three in-frame deletions. Six patients had homozygous mutations, all except one non-R882 missense substitutions, and six patients had two mutations, all non-R882. Therefore, of the 272 DNMT3A ${ }^{\text {MUT }}$ patients, 175 (64\%) had R882 mutations, 59 (22\%) had non-R882 missense mutations, 35 (13\%) had truncations or in-frame deletions, and three $(1 \%)$ had two mutations of differing types.

The median DNMT3A mutant level for 172 patients with $\mathrm{R} 882 \mathrm{H}$ $(\mathrm{n}=123)$ or $\mathrm{R} 882 \mathrm{C}(\mathrm{n}=49)$ mutations was $47 \%$ (range, $15 \%$ to $85 \%$; Appendix Fig A1, online only), indicative of a heterozygous mutation in nearly all cells. NPM1 ${ }^{\mathrm{MUT}}$ levels were available from 147 of these 


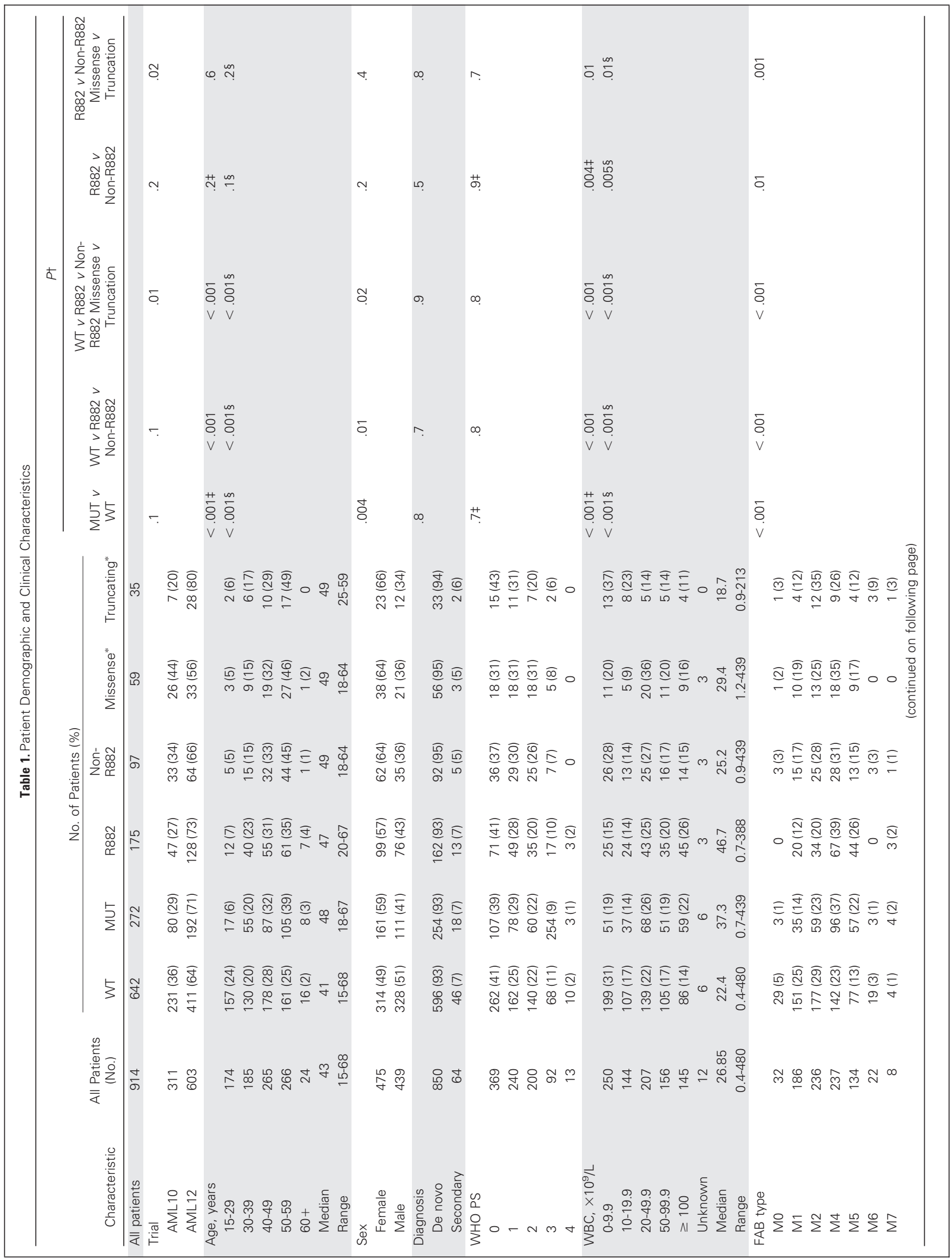




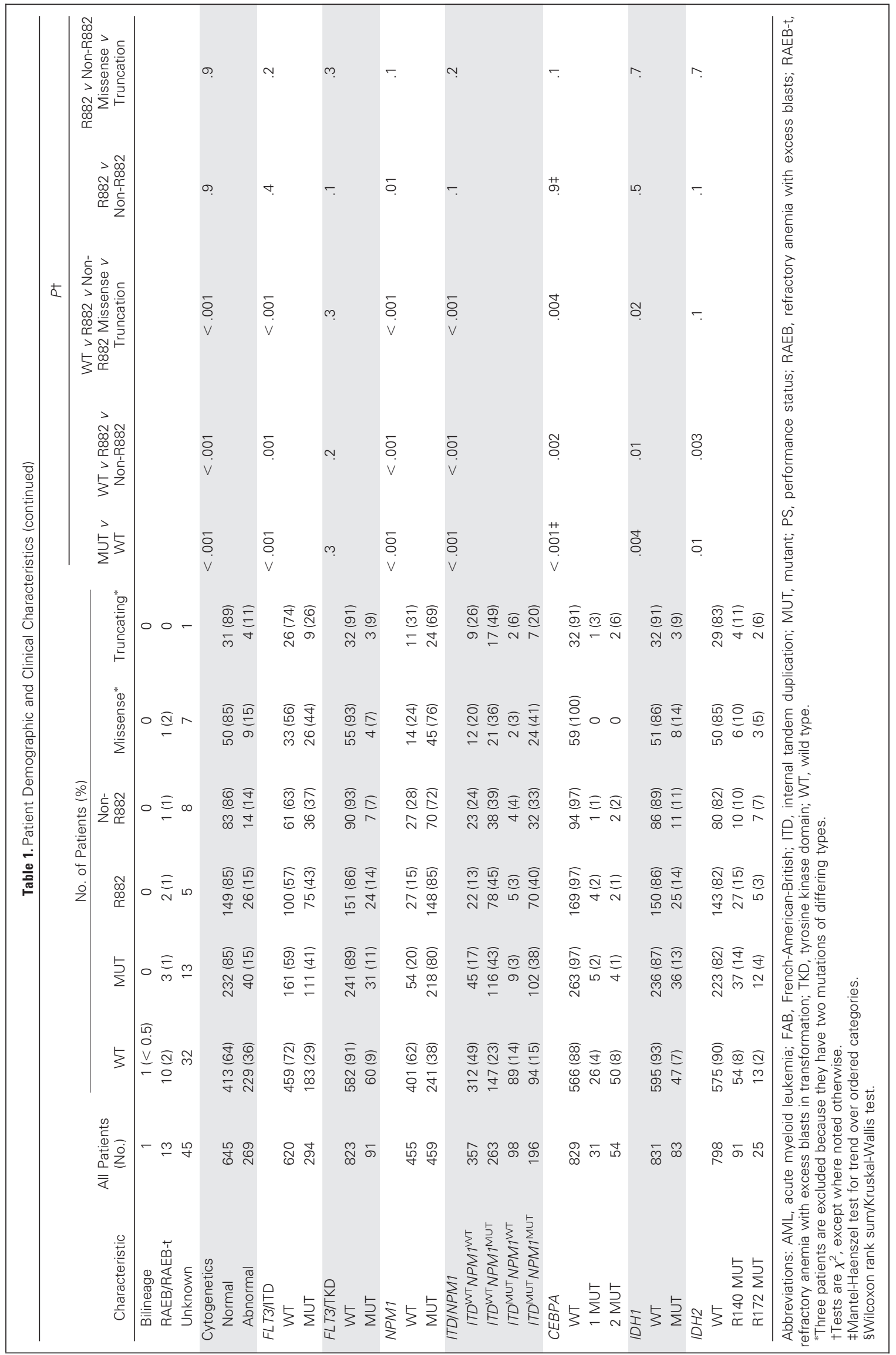




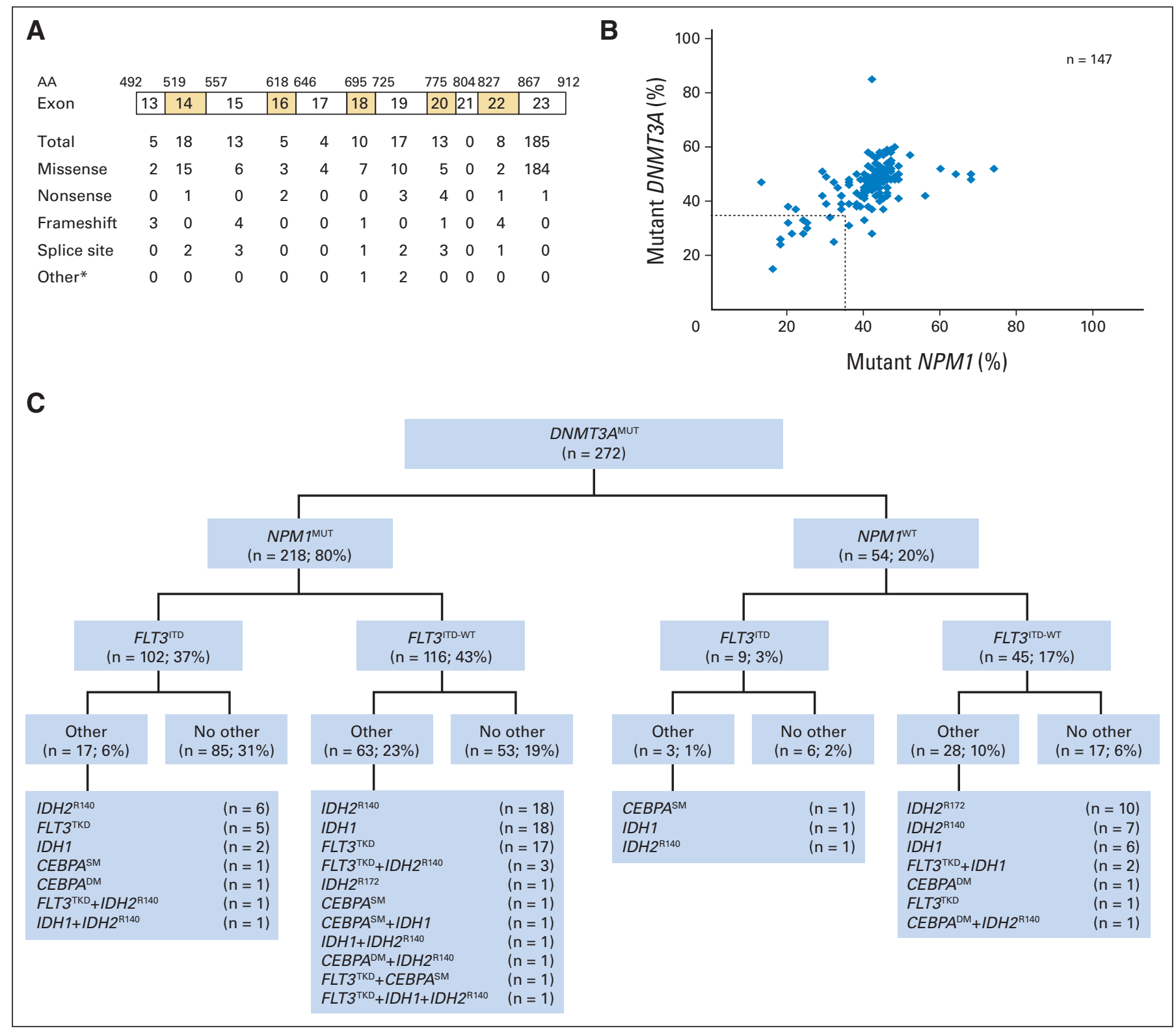

Fig 1. DNMT3A mutations detected: mutant type, level, and coincidence with other recurrent mutations. (A) Exonic location and the mutant type of all detected mutations. The amino acid (AA) given is the first AA of the exon, except for the last AA (912). (*) In-frame deletions. (B) Comparison of DNMT3A and NPM1 mutant levels in 147 patients with R882H or R882C mutations. The dotted line indicates a mutant level of $35 \%$. (C) Coincidence of other mutations in the cohort of 272 DNMT3A-mutated patients. DM, double mutant; ITD, internal tandem duplication; SM, single mutant; TKD, tyrosine kinase domain; WT, wild type.

patients, and of the 12 patients with a DNMT3A ${ }^{\mathrm{MUT}}$ level less than $35 \%$, nine also had an NPM1 ${ }^{\text {MUT }}$ level less than 35\% (Fig 1B). Assuming that an NPM1 mutation is found in virtually all leukemic cells, this suggests that the instances of low-level $D N M T 3 A^{\mathrm{MUT}}$ were a result of nonleukemic cell contamination rather than the mutation being present only in a subclone of leukemic cells.

\section{Patient Characteristics According to DNMT3A Genotype}

$D N M T 3 A^{\text {MUT }}$ patients were significantly older than $D N M T 3 A^{\text {WT }}$ patients $(P<.001)$ and more likely to be female $(P=.004)$ and have a higher presenting WBC $(P<.001$; Table 1$)$. There was a significant difference across morphologic subgroups $(P<.001)$, with higher rates in
M4 (40\%) and M5 (43\%), which represented 37\% and 22\%, respectively, of all $D N M T 3 A^{\mathrm{MUT}}$ patients. DNMT3A $A^{\mathrm{MUT}}$ patients were significantly more likely than $D N M T 3 A^{\mathrm{WT}}$ patients to have an NK rather than an IR abnormal karyotype $(P<.001)$. Coincidence with other recurrent mutations showed a positive correlation between $D N M T 3 A^{\mathrm{MUT}}$ and $N P M 1^{\mathrm{MUT}}(P<.001)$, FLT3 $^{\mathrm{ITD}}(P<.001)$, IDH1 $^{\mathrm{MUT}}(P=.004)$, and $\mathrm{IDH} 2^{\mathrm{MUT}}(P=.01)$, but a negative correlation with $C E B P A^{\mathrm{MUT}}(P<$ .001 ; Table 1; Fig 1C). Of note, $80 \%$ of the DNMT3A ${ }^{\mathrm{MUT}}$ samples were $N P M 1^{\mathrm{MUT}}, 37 \%$ were $F L T 3^{\mathrm{ITD}} N P M 1^{\mathrm{MUT}}$, and $43 \%$ were FLT3 ${ }^{\text {ITD-WT }}{ }^{N P M 1}{ }^{\text {MUT }}$. Conversely, $47 \%$ of $N P M 1^{\text {MUT }}$ samples were DNMT3A ${ }^{\mathrm{MUT}}$.

There was no difference in age, sex, and type of leukemia between $D N M T 3 A^{\mathrm{MUT}}$ patients with R882 (DNMT3A $\left.{ }^{\mathrm{R} 882}\right)$ and 


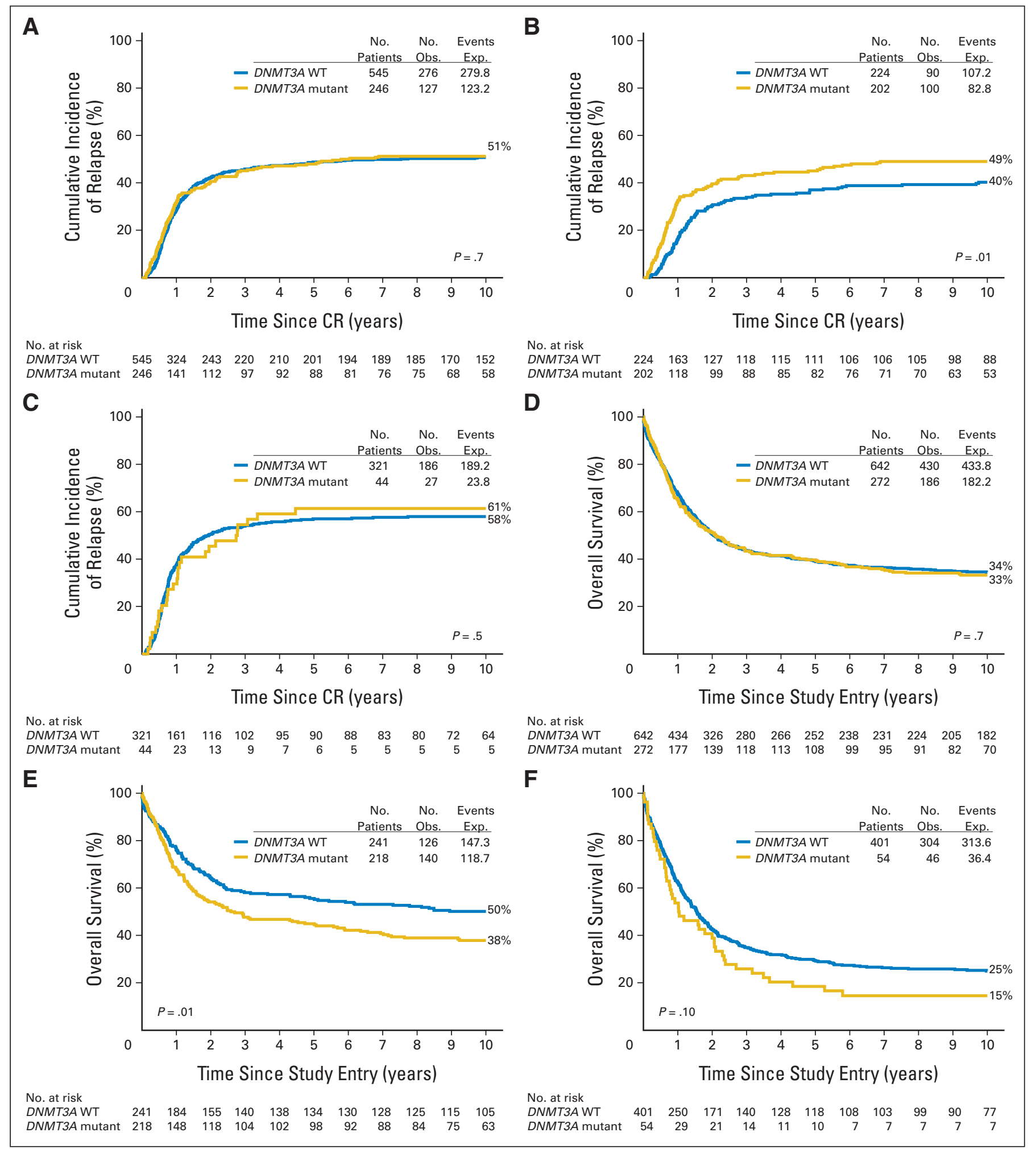

Fig 2. Kaplan-Meier curves for outcome stratified according to DNMT3A genotype. Cumulative incidence of relapse in (A) the total cohort, (B) NPM1MUT patients, and (C) NPM1 ${ }^{\mathrm{WT}}$ patients. Overall survival in (D) the total cohort, (E) NPM1 $1^{\mathrm{MUT}}$ patients, and (F) NPM1 ${ }^{\mathrm{WT} T}$ patients. CR, complete remission; Exp., expected; MUT, mutation; Obs., observed; WT, wild type.

DNMT3A $A^{\text {MUT }}$ patients with other mutations (DNMT3A ${ }^{\text {non-R882 }}$, irrespective of the type of non-R882 mutation (Table 1). However, $D N M T 3 A^{\mathrm{R} 882}$ patients had a significantly higher WBC than DNMT3A $A^{\text {non-R82 }}$ patients $(P=.005)$ and a significantly higher correlation with $N P M 1^{\mathrm{MUT}}(P=.01)$. In the $D N M T 3 A^{\text {non-R882 }}$ patients, those with missense mutations had a higher median WBC and more coincidence with $N P M 1^{\mathrm{MUT}}, \mathrm{FLT}^{\mathrm{ITD}}$, and $\mathrm{IDH} 1^{\mathrm{MUT}}$ than those with truncations (Table 1). 


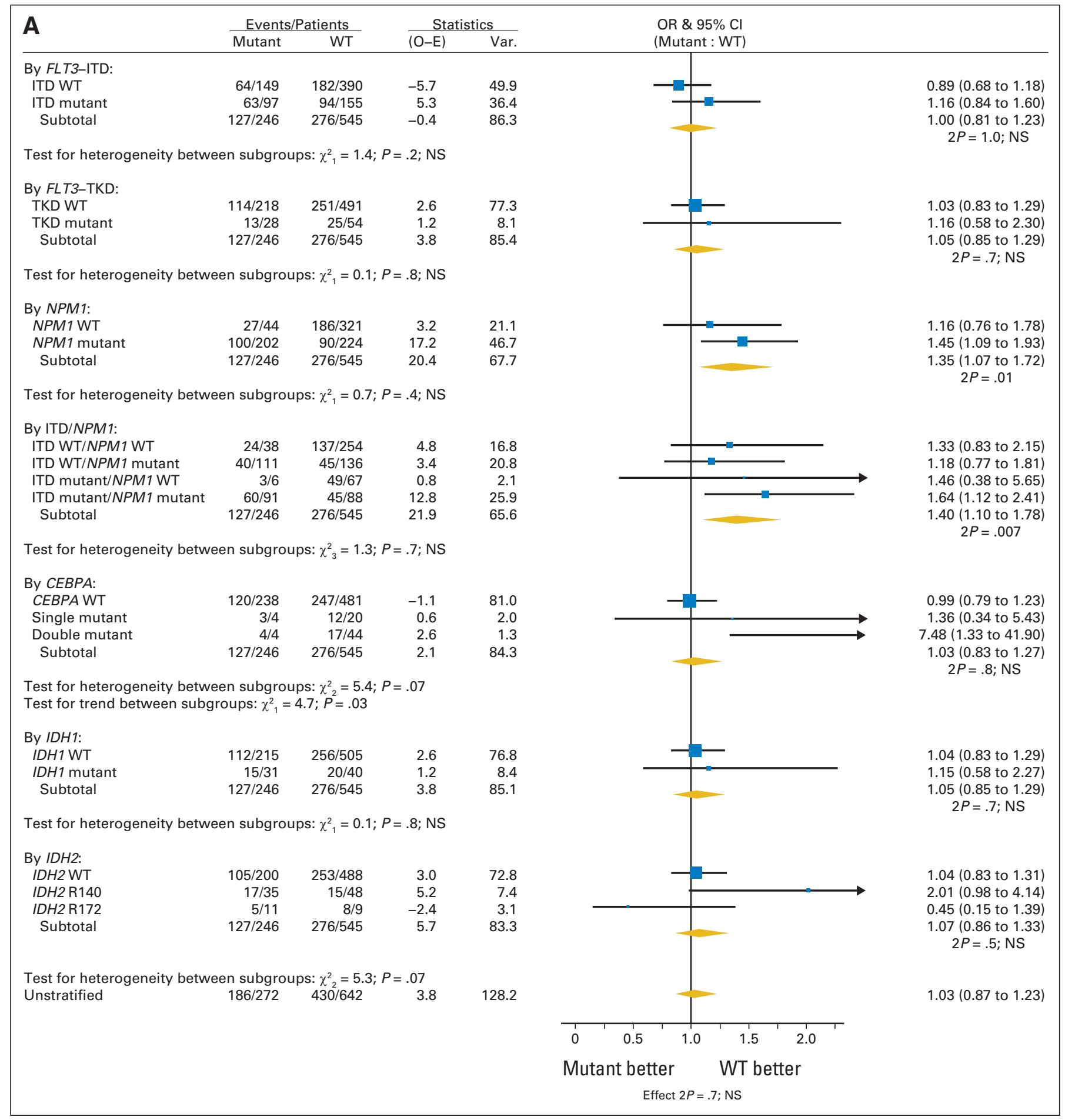

Fig 3. Forest plots for the impact of a DNMT3A mutation on outcome in different molecular groups. (A) Relapse. (B) Overall survival. E, expected; ITD, internal tandem duplication; NS, not significant; O, observed; OR, odds ratio; TKD, tyrosine kinase domain; WT, wild type.

\section{Response to Therapy and Long-Term Outcome Stratified According to DNMT3A Genotype}

There was a slightly but significantly higher remission rate in $D N M T 3 A^{\mathrm{MUT}}$ patients than $D N M T 3 A^{\mathrm{WT}}$ patients $(90 \% v 85 \%$, respectively; $P=.03$ ). However, this can probably be attributed to the association with $N P M 1^{\mathrm{MUT}}$, and the significance was not maintained in multivariable analysis taking into account patient characteristics and other molecular markers (odds ratio, $0.74 ; 95 \% \mathrm{CI}, 0.43$ to 1.28 ; $P=.3$; Appendix Table A4, online only). In the total group, neither CIR nor OS differed according to $D N M T 3 A$ genotype $(P=.7$ for both; Figs $2 \mathrm{~A}$ and $2 \mathrm{D}$ ). However, these results suggesting that $D N M T 3 A$ mutations are not associated with a poor prognosis must be interpreted with 


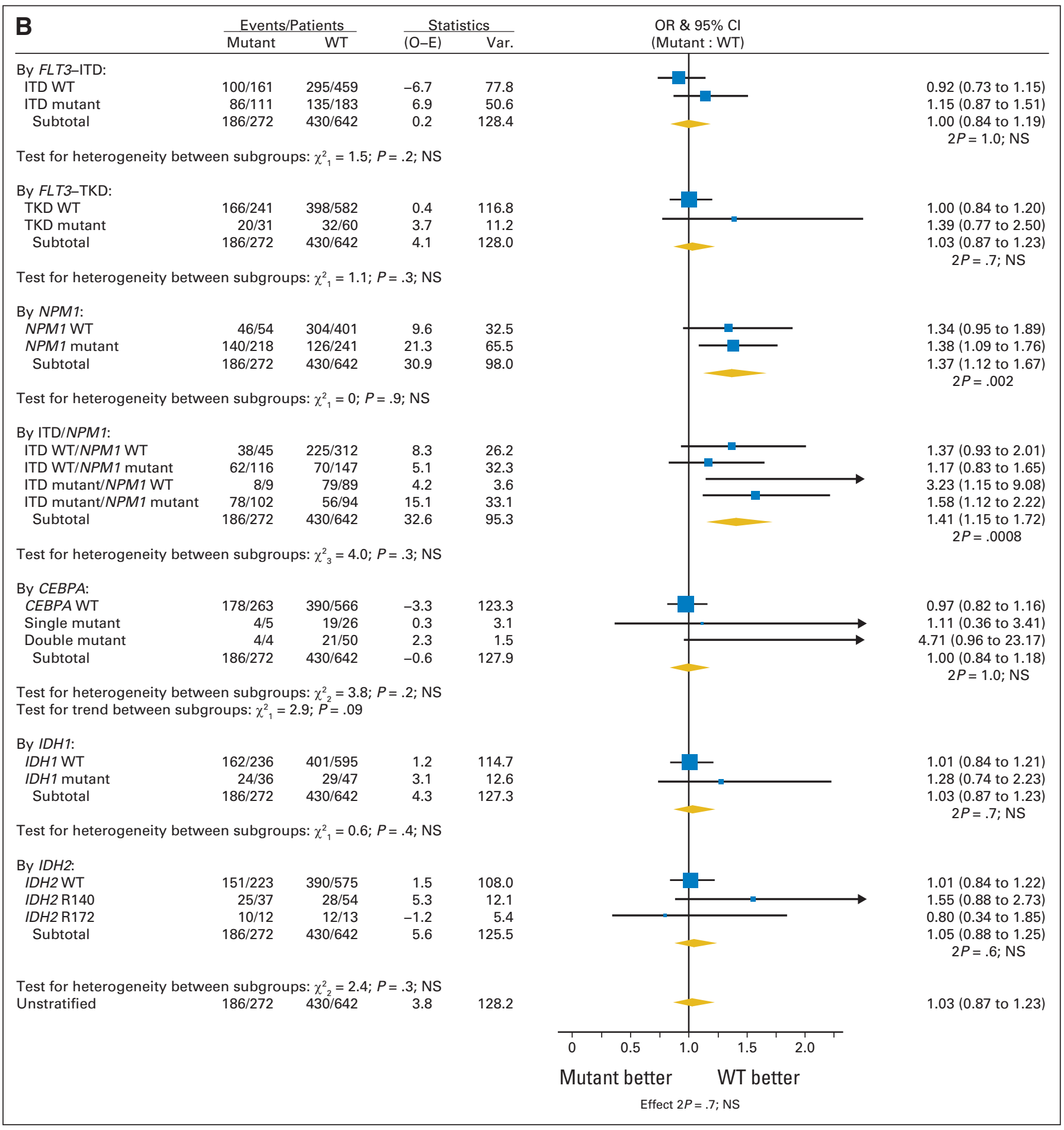

Fig 3. (continued).

caution because of the high level of concordance with $N P M 1^{\mathrm{MUT}}$, which is associated with a good prognosis. Therefore, we performed a subgroup analysis of $N P M 1^{\mathrm{MUT}}$ and $N P M 1^{\mathrm{WT}}$ patients.

The remission rate in $N P M 1^{\mathrm{MUT}}$ patients was $93 \%$ for both $D N M T 3 A^{\mathrm{MUT}}$ and $D N M T 3 A^{\mathrm{WT}}$; in $N P M 1^{\mathrm{WT}}$ patients, the remission rates were $81 \%$ and $80 \%$ for $D N M T 3 A^{\mathrm{MUT}}$ and $D N M T 3 A^{\mathrm{WT}}$, respectively. $D N M T 3 A^{\mathrm{MUT}}$ was associated with higher CIR in both
$N P M 1^{\mathrm{MUT}}$ and $N P M 1^{\mathrm{WT}}$ patients when analyzed separately (Figs $2 \mathrm{~B}$ and $2 \mathrm{C})$. Although this was not significant for the $N P M 1^{\text {WT }}$ $D N M T 3 A^{\mathrm{MUT}}$ patients, in the analysis stratified for NPM1 genotype, the overall impact of $D N M T 3 A^{\mathrm{MUT}}$ was significantly adverse $(\mathrm{HR}$, 1.35 ; $95 \% \mathrm{CI}, 1.07$ to $1.72 ; P=.01$ ), and testing for heterogeneity showed that the impact did not significantly differ between $N P M 1^{\text {MUT }}$ and $N P M 1^{\mathrm{WT}}$ patients $(P=.4$; Fig $3 \mathrm{~A})$. There was no difference in the 


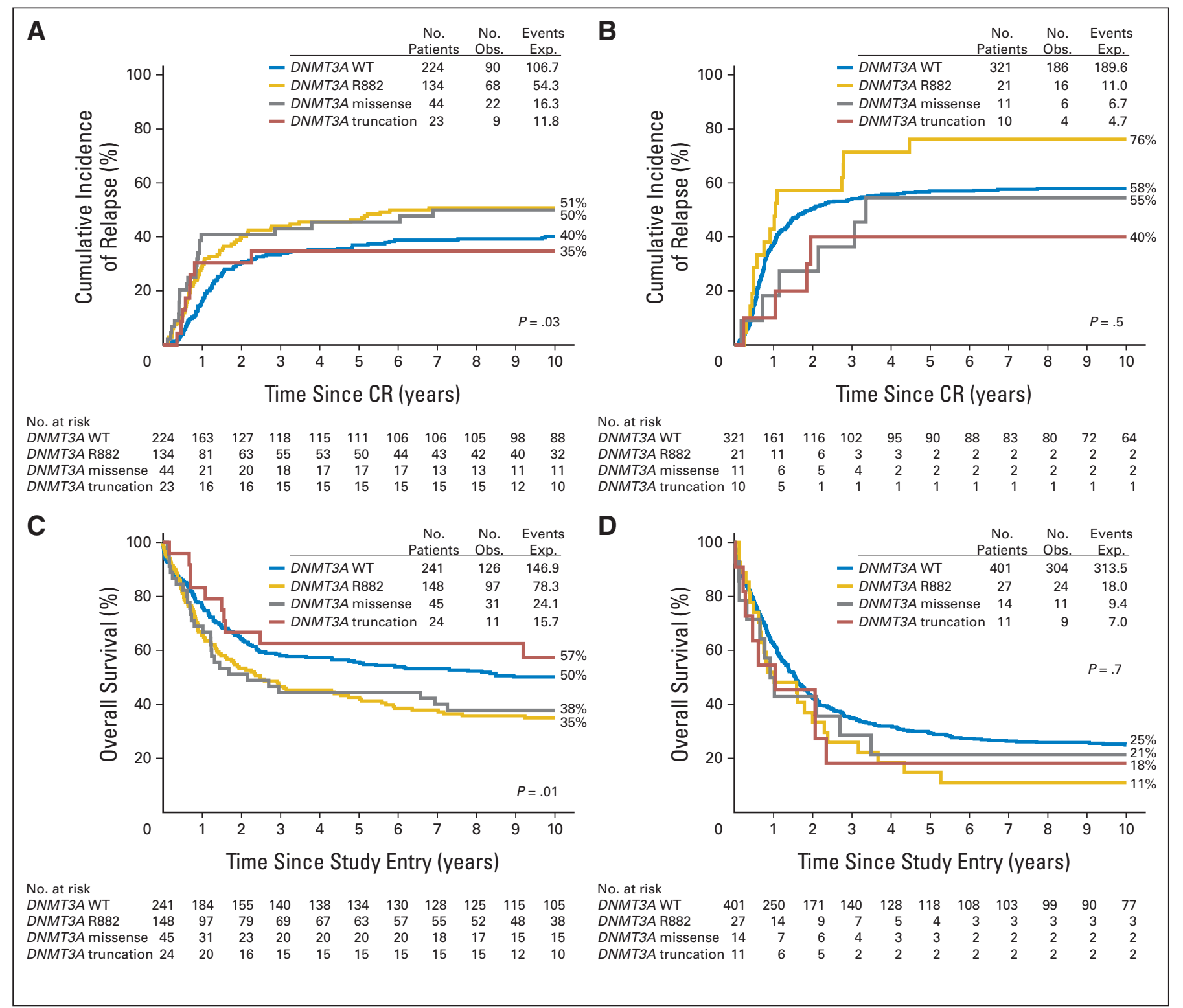

Fig 4. Kaplan-Meier curves for outcome stratified according to type of DNMT3A mutation. Cumulative incidence of relapse in (A) NPM1 ${ }^{\mathrm{MUT}}$ patients and (B) NPM1 ${ }^{\mathrm{WT}}$ patients. Overall survival in (C) NPM1 ${ }^{\mathrm{MUT}}$ patients and (D) NPM1 ${ }^{\mathrm{WT}}$ patients. CR, complete remission; Exp., expected; Obs., observed; WT, wild type.

impact stratified according to FLT3 ${ }^{\mathrm{ITD}}$ status. Further analysis of the four subgroups defined by NPM1 and FLT3 ${ }^{\mathrm{TTD}}$ genotype similarly showed no heterogeneity between the groups of the poor prognostic impact on CIR associated with DNMT3A ${ }^{\mathrm{MUT}}(P=.7$; Fig 3A). In accord with these results, $D N M T 3 A^{\mathrm{MUT}}$ patients had worse OS than $D N M T 3 A^{\mathrm{WT}}$ patients with either $N P M 1^{\mathrm{MUT}}$ or $N P M 1^{\mathrm{WT}}$ genotype (Figs $2 \mathrm{E}$ and $2 \mathrm{~F}$ ), and although not significant for the $N P M 1^{\mathrm{WT}}$ $D N M T 3 A^{\mathrm{MUT}}$ patients, the overall impact in the stratified analysis was highly significant (HR, 1.37; 95\% CI, 1.12 to 1.87 ; $P=.002$; Fig 3B). There was no evidence of heterogeneity of the impact of a DNMT3A mutation between $N P M 1^{\mathrm{MUT}}$ and $N P M 1^{\mathrm{WT}}$ patients $(P=.9)$ or between the four NPM1/FLT3 ${ }^{\mathrm{TTD}}$ genotypes $(P=.3$; Fig $3 \mathrm{~B})$. Of note, four of the 54 patients with double $C E B P A$ mutations $\left(C E B P A^{\mathrm{DM}}\right)$ were also $D N M T 3 A^{\mathrm{MUT}}$. All four patients attained remission but experienced relapse, which represents a significantly higher CIR com- pared with $C E B P A^{\mathrm{DM}} D N M T 3 A^{\mathrm{WT}}$ patients $(P=.03$; Fig $3 \mathrm{~A})$. There was also a trend to lower OS in these $C E B P A^{\mathrm{DM}} D N M T 3 A^{\mathrm{MUT}}$ patients $(P=.1$; Fig 3B $)$.

In multivariable analysis, $D N M T 3 A^{\mathrm{MUT}}$ was a significant adverse risk factor for CIR (HR, 1.27; 95\% CI, 1.01 to $1.61 ; P=.04)$ and a borderline adverse factor for OS (HR, 1.19; 95\% CI, 0.98 to 1.45; $P=$ .1; Appendix Table A4). In a forward selection model considering all clinically relevant factors, $D N M T 3 A$ genotype entered as a significant factor for relapse but not complete remission or OS (Appendix Table A5, online only).

\section{Outcome Stratified According to Type of DNMT3A Mutation}

To assess the impact of different DNMT3A mutations, outcome was considered according to the type of mutation $-\mathrm{R} 882$, non-R882 


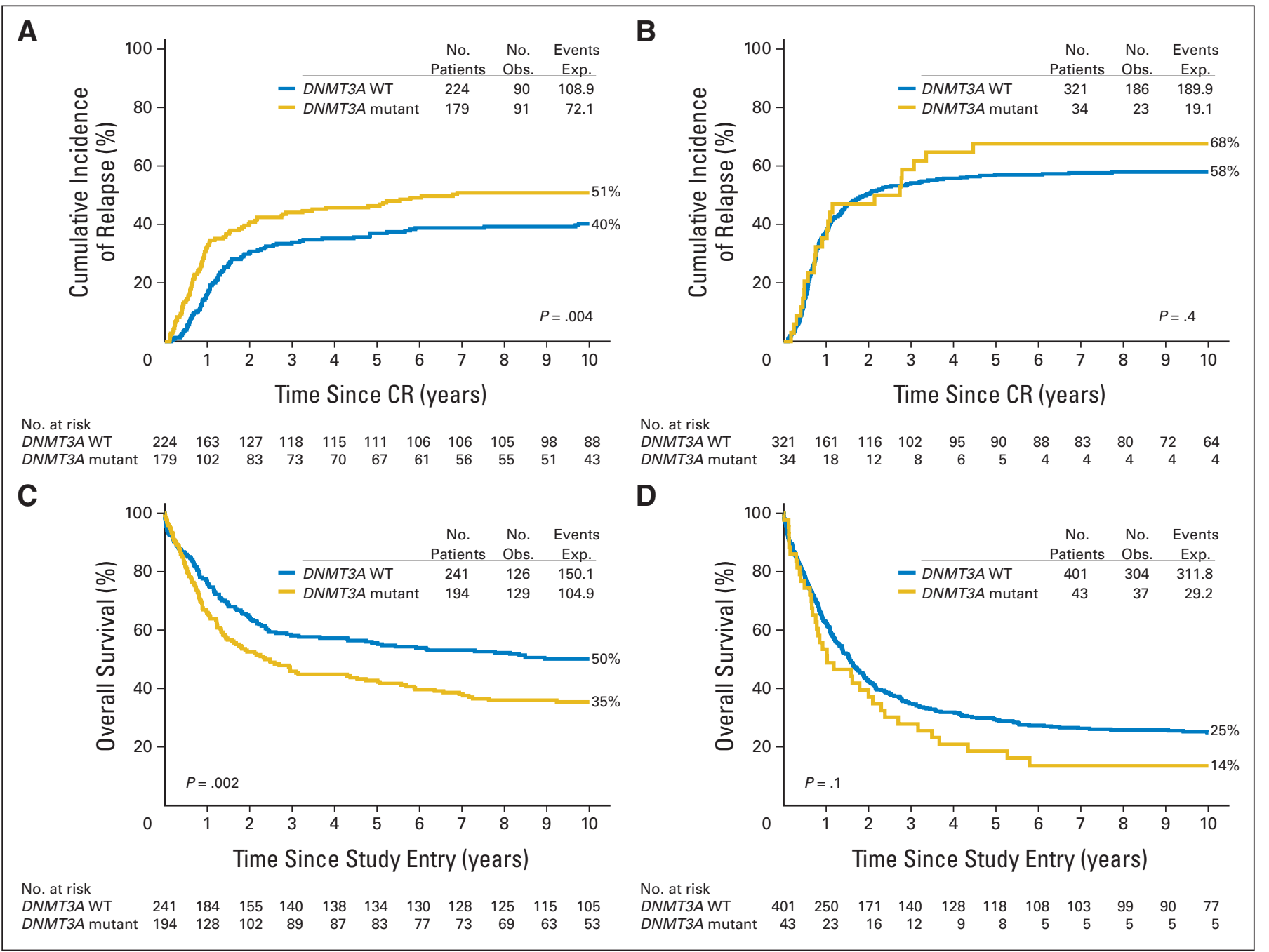

Fig 5. Kaplan-Meier curves for outcome stratified according to DNMT3A genotype excluding truncation mutations. Cumulative incidence of relapse in (A) NPM1MUT patients and (B) NPM1 ${ }^{W T}$ patients. Overall survival in (C) NPM1 $1^{M U T}$ patients and (D) NPM1 ${ }^{W T}$ patients. CR, complete remission; Exp., expected; Obs., observed; WT, wild type.

missense, or truncation. Three patients were excluded from the analysis because they had two mutations of differing types.

The remission rate did not differ significantly according to mutation type ( $P=.4$ across the three groups) in either $N P M 1^{\mathrm{MUT}}(P=$ .2) or $N P M 1^{\mathrm{WT}}(P=.6)$ patients. CIR was higher in $D N M T 3 A^{\mathrm{R} 882}$ patients than $D N M T 3 A^{\mathrm{WT}}$ patients with either $N P M 1^{\mathrm{MUT}}$ or $N P M 1^{\text {WT }}$ genotype, whereas patients with truncations had similar CIR to those with $D N M T 3 A^{\mathrm{WT}}$ among $N P M 1^{\mathrm{MUT}}$ patients and lower CIR than those with $D N M T 3 A^{\mathrm{WT}}$ among $N P M 1^{\mathrm{WT}}$ patients (Figs $4 \mathrm{~A}$ and $4 \mathrm{~B})$. Non-R882 missense mutations were associated with higher CIR than $D N M T 3 A^{\mathrm{WT}}$ in $N P M 1^{\mathrm{MUT}}$ patients, equivalent to $D N M T 3 A^{\mathrm{R} 882}$ patients, and similar CIR to DNMT3A ${ }^{\mathrm{WT}}$ in $N P M 1^{\mathrm{WT}}$ patients. In accord with this, patients with $D N M T 3 A^{\mathrm{R} 882}$ had worse OS than patients with $D N M T 3 A^{\mathrm{WT}}$ and either $N P M 1^{\mathrm{MUT}}$ or $N P M 1^{\mathrm{WT}}$ genotype (Figs 4C and 4D). Truncations were associated with nonsignificantly better $\mathrm{OS}$ in $N P M 1^{\mathrm{MUT}}$ patients and were similar to $D N M T 3 A^{\mathrm{WT}}$ in $N P M 1^{\mathrm{WT}}$ patients. Non-R882 missense mutations were associated with poorer OS in $N P M 1^{\mathrm{MUT}}$ patients, similar to R882 mutations, but seemed to have a lesser impact in $N P M 1^{\mathrm{WT}}$ patients, although there were only 12 patients in this category.
Because these results suggested that DNMT3A truncation and missense mutations may differ in their impact on outcome, the data were reanalyzed excluding patients with truncations. The results were comparable to the initial results, but the differences were of slightly greater significance (Figs 5A to 5D).

\section{DISCUSSION}

In this large cohort of cytogenetically IR younger patients with AML, a DNMT3A mutation was associated with poorer prognosis, but this difference was only observed if the results were analyzed separately according to NPM1 genotype, where worse OS was seen in $D N M T 3 A^{\mathrm{MUT}}$ patients with both $N P M 1^{\mathrm{MUT}}$ and $N P M 1^{\mathrm{WT}}$ genotype. This is an example of Simpson's paradox. ${ }^{36}$ It results from the strong association between DNMT3A and NPM1 mutations and the opposing prognostic impact of the two mutants. In our cohort, $80 \%$ of $D N M T 3 A^{\mathrm{MUT}}$ patients were also $N P M 1^{\mathrm{MUT}}$, which leads to a marked inequality in the proportion of DNMT3A ${ }^{\mathrm{MUT}}$ in the $N P M 1^{\mathrm{MUT}}$ and $N P M 1^{\text {WT }}$ patients. Because outcome of the total group reflects the 
relative proportion of the different genotypic subgroups, the effect that is seen separately in the groups is masked when data from the two groups are combined. This is illustrated in Appendix Table A6 (online only). This paradox may explain, at least in part, some of the differences reported to date in studies examining the prognostic impact of DNMT3A mutations, in particular where the genotypic subgroups are small and likely to be less statistically robust. ${ }^{3-7,9}$ Although there was a significant association between DNMT3A ${ }^{\text {MUT }}$ and $F L T 3^{\text {ITD }}$ (41\% of $D N M T 3 A^{\mathrm{MUT}}$ patients were $F L T 3^{\mathrm{ITD}}$ ), no such differences were observed when $D N M T 3 A^{\text {MUT }}$ patients were stratified according to $F_{T T 3}{ }^{\mathrm{ITD}}$ status because both are associated with poorer outcome.

Studies have shown that different mutations within the same gene may be associated with variable patient characteristics and outcome (eg, IDH2 R140 and R172 mutations). ${ }^{32,37}$ Therefore, consideration needs to be given to the variety of mutations identified in the DNMT3A gene and their potentially differing functional consequences. In our cohort, $64 \%$ of $D N M T 3 A^{\mathrm{MUT}}$ patients had a mutation at residue $\mathrm{R} 882$, comparable to the incidence reported in other studies (mean, 68\%; range, 60\% to $83 \%$; test for heterogeneity, $P=.7$ ). ${ }^{2,4-9,38}$ However, the remaining mutations were scattered across the zinc finger and methyltransferase domains and included not only other missense mutations ( $22 \%$ of mutated patients), but also truncations (13\%). Analysis in the three mutant categories suggested that, whereas R882 and non-R882 missense mutations were associated with a similar adverse outcome, the truncations seemed to have a different functional impact on the R882 mutations and should be considered as equivalent to DNMT3 $A^{\mathrm{WT}}$ from a prognostic standpoint. These data require corroboration from other large cohorts, because there were only 35 patients with a truncation, which therefore results in limited statistical power when split into the $N P M 1^{\mathrm{MUT}}$ and $N P M 1^{\mathrm{WT}}$ groups. If total loss of protein from the mutated allele leads to haploinsufficiency, then the data are compatible with the lack of impact on hematopoiesis observed for Dnmt3a loss in mouse models, ${ }^{24-26}$ rather than the dominantnegative effect that has been demonstrated for the R882 mutant. ${ }^{20}$ However, it does raise the issue of the role of such mutations in leukemogenesis, and further studies will be required to investigate this.

For prognostication purposes, combined analysis of the patients with non-R882 missense mutations suggested that they should be considered together with R882-mutated patients as having worse outcome than DNMT3A ${ }^{\mathrm{WT}}$ patients. However, most are unique, and they comprise a highly heterogeneous group of mutations that disrupt different domains of the enzyme. Although both the SIFT and PolyPhen algorithms predict that the majority of mutations in the zinc finger and methyltransferase domains are likely to be deleterious, there is evidence that they differ in their functional and cellular signif- icance. For example, G543 was the second most commonly mutated amino acid, detected in 12 patients (4\%). It is close to the histone $\mathrm{H} 3 \mathrm{~K} 4$ binding surface and does not affect DNA methylation activity but has increased in vitro ability to interact with histone H3. ${ }^{18}$ Similarly, differences in mean methylation levels and methylated CpGs have been reported between patients with R882 and non-R882 mutations. ${ }^{21}$ More detailed structural and functional analysis of these mutants may thus provide information about the normal roles of DNMT3A and its interactions with other proteins and how disruption of these processes may lead to AML.

From a therapeutic viewpoint, our data confirm that $D_{N M T} A^{\mathrm{MUT}}$ should be treated as a poor-risk factor. This is of particular relevance to management of patients currently considered as favorable risk, in particular those with $C E B P A^{\mathrm{DM}}$, where $D N M T 3 A^{\text {MUT }}$ patients were significantly more likely to experience relapse, and those with $N P M 1^{\mathrm{MUT}} F L T 3^{\mathrm{ITD}-\mathrm{WT}}$, where the lack of heterogeneity between subgroups indicated that these patients should not be considered differently from the other NPM1/FLT3 genotype groups. The adverse outcome associated with the non-R882 missense mutations also indicates that screening strategies cannot be limited to analysis of exon 23 alone. It is important to note that the need for subgroup analysis in prognostication, as in this case, requires cautious interpretation of the data because of the pitfalls of multiple comparisons and potential bias. The controversy over determining how mutant status should be used for risk stratification, even for the three most commonly mutated genes (DNMT3A, NPM1, and FLT3), reflects the increasing challenge for determining outcome analysis in the face of the extensive genotypic heterogeneity and variable interaction between coincident mutations that is becoming apparent in AML.

\section{AUTHORS' DISCLOSURES OF POTENTIAL CONFLICTS OF INTEREST}

Disclosures provided by the authors are available with this article at www.jco.org.

\section{AUTHOR CONTRIBUTIONS}

Conception and design: Rosemary E. Gale, David C. Linch

Financial support: Rosemary E. Gale, David C. Linch

Provision of study materials or patients: Alan K. Burnett

Collection and assembly of data: Katarina Lamb, Christopher Allen,

Dima El-Sharkawi, Cassandra Stowe, Sarah Jenkinson, Steven Tinsley,

Glenda Dickson, Alan K. Burnett

Data analysis and interpretation: Rosemary E. Gale, Robert K. Hills, David C. Linch

Manuscript writing: All authors

Final approval of manuscript: All authors

\section{REFERENGES}

1. Cancer Genome Atlas Research Network: Genomic and epigenomic landscapes of adult de novo acute myeloid leukemia. $N$ Engl J Med 368:2059-2074, 2013

2. Ley $T J$, Ding $L$, Walter MJ, et al: DNMT3A mutations in acute myeloid leukemia. N Engl J Med 363:2424-2433, 2010

3. Shen $Y$, Zhu YM, Fan X, et al: Gene mutation patterns and their prognostic impact in a cohort of
1185 patients with acute myeloid leukemia. Blood 118:5593-5603, 2011

4. Thol F, Damm F, Lüdeking A, et al: Incidence and prognostic influence of DNMT3A mutations in acute myeloid leukemia. J Clin Oncol 29:2889-2896, 2011

5. Ribeiro AF, Pratcorona M, Erpelinck-Verschueren C, et al: Mutant DNMT3A: A marker of poor prognosis in acute myeloid leukemia. Blood 119:58245831, 2012

6. Renneville A, Boissel N, Nibourel O, et al: Prognostic significance of DNA methyltransferase 3A mutations in cytogenetically normal acute my- eloid leukemia: A study by the Acute Leukemia French Association. Leukemia 26:1247-1254, 2012

7. Hou HA, Kuo YY, Liu CY, et al: DNMT3A mutations in acute myeloid leukemia: Stability during disease evolution and clinical implications. Blood 119:559-568, 2012

8. Marcucci G, Metzeler KH, Schwind S, et al: Age-related prognostic impact of different types of DNMT3A mutations in adults with primary cytogenetically normal acute myeloid leukemia. J Clin Oncol 30:742-750, 2012 
9. Gaidzik VI, Schlenk RF, Paschka $P$, et al: Clinical impact of DNMT3A mutations in younger adult patients with acute myeloid leukemia: Results of the AML Study Group (AMLSG). Blood 121:47694777, 2013

10. Marcucci G, Haferlach T, Döhner H: Molecular genetics of adult acute myeloid leukemia: Prognostic and therapeutic implications. J Clin Oncol 29:475486, 2011

11. Ofran Y, Rowe JM: Genetic profiling in acute myeloid leukaemia: Where are we and what is its role in patient management? $\mathrm{Br} \mathrm{J}$ Haematol 160 303-320, 2013

12. Cornelissen JJ, Gratwohl A, Schlenk RF, et al: The European LeukemiaNet AML Working Party consensus statement on allogeneic HSCT for patients with $\mathrm{AML}$ in remission: An integrated-risk adapted approach. Nat Rev Clin Oncol 9:579-590, 2012

13. O'Donnell MR, Abboud CN, Altman J, et al: Acute myeloid leukemia. J Natl Compr Canc Netw 10:984-1021, 2012

14. Shivarov V, Gueorguieva R, Stoimenov A, et al: DNMT3A mutation is a poor prognosis biomarker in AML: Results of a meta-analysis of $4500 \mathrm{AML}$ patients. Leuk Res 37:1445-1450, 2013

15. Tie $\mathrm{R}$, Zhang $\mathrm{T}, \mathrm{Fu} \mathrm{H}$, et al: Association between DNMT3A mutations and prognosis of adults with de novo acute myeloid leukemia: A systematic review and meta-analysis. PloS One 9:e93353, 2014

16. Li KK, Luo LF, Shen Y, et al: DNA methyltransferases in hematologic malignancies. Semin Hematol 50:48-60, 2013

17. Yamashita $Y$, Yuan J, Suetake I, et al: Arraybased genomic resequencing of human leukemia Oncogene 29:3723-3731, 2010

18. Yan $X J, X u J, G u Z H$, et al: Exome sequencing identifies somatic mutations of DNA methyltransferase gene DNMT3A in acute monocytic leukemia. Nat Genet 43:309-315, 2011

19. Holz-Schietinger C, Matje DM, Reich NO: Mutations in DNA methyltransferase (DNMT3A) observed in acute myeloid leukemia patients disrupt processive methylation. J Biol Chem 287:3094130951, 2012

20. Kim SJ, Zhao H, Hardikar S, et al: A DNMT3A mutation common in AML exhibits dominantnegative effects in murine ES cells. Blood 122:40864089, 2013

21. Russler-Germain DA, Spencer DH, Young MA, et al: The R882H DNMT3A mutation associated with AML dominantly inhibits wild-type DNMT3A by blocking its ability to form active tetramers. Cancer Cell 25:442-454, 2014

22. Holz-Schietinger C, Matje DM, Harrison MF et al: Oligomerization of DNMT3A controls the mechanism of de novo DNA methylation. J Biol Chem 286:41479-41488, 2011

23. Kervestin S, Jacobson A: NMD: A multifaceted response to premature translational termination. Nat Rev Mol Cell Biol 13:700-712, 2012

24. Okano M, Bell DW, Haber DA, et al: DNA methyltransferases Dnmt3a and Dnmt3b are essential for de novo methylation and mammalian development. Cell 99:247-257, 1999

25. Tadokoro $\mathrm{Y}$, Ema H, Okano $\mathrm{M}$, et al: De novo DNA methyltransferase is essential for self-renewal, but not for differentiation, in hematopoietic stem cells. J Exp Med 204:715-722, 2007

26. Challen GA, Sun D, Jeong $M$, et al: Dnmt3a is essential for hematopoietic stem cell differentiation. Nat Genet 44:23-31, 2012

27. Grimwade D, Hills RK, Moorman AV, et al: Refinement of cytogenetic classification in acute myeloid leukemia: Determination of prognostic significance of rare recurring chromosomal abnormalities among 5876 younger adult patients treated in the United Kingdom Medical Research Council trials. Blood 116:354-365, 2010

28. Gale RE, Green C, Allen $C$, et al: The impact of FLT3 internal tandem duplication mutant level, number, size, and interaction with NPM1 mutations in a large cohort of young adult patients with acute myeloid leukemia. Blood 111:2776-2784, 2008

29. Mead AJ, Linch DC, Hills RK, et al: FLT3 tyrosine kinase domain mutations are biologically distinct from and have a significantly more favorable prognosis than FLT3 internal tandem duplications in patients with acute myeloid leukemia. Blood 110 1262-1270, 2007

30. Green CL, Koo KK, Hills RK, et al: Prognostic significance of CEBPA mutations in a large cohort of younger adult patients with acute myeloid leukemia: Impact of double CEBPA mutations and the interaction with FLT3 and NPM1 mutations. J Clin Oncol 28:2739-2747, 2010

31. Green $C L$, Evans $C M$, Hills $R K$, et al: The prognostic significance of $\mathrm{IDH} 1$ mutations in younger adult patients with acute myeloid leukemia is dependent on FLT3/ITD status. Blood 116:27792782, 2010

32. Green $C L$, Evans $C M$, Zhao $L$, et al: The prognostic significance of IDH2 mutations in $\mathrm{AML}$ depends on the location of the mutation. Blood 118:409-412, 2011

33. Hann IM, Stevens RF, Goldstone $A H$, et al: Randomized comparison of DAT versus ADE as induction chemotherapy in children and younger adults with acute myeloid leukemia: Results of the Medical Research Council's 10th AML trial (MRC AML10). Blood 89:2311-2318, 1997

34. Burnett AK, Hills RK, Milligan DW, et al: Attempts to optimize induction and consolidation treatment in acute myeloid leukemia: Results of the MRC AML12 trial. J Clin Oncol 28:586-595, 2010

35. Early Breast Cancer Trialists Collaborative Group: Treatment of Early Breast Cancer Volume I: Worldwide Evidence 1985-90. Oxford, United Kingdom, Oxford University Press, 1990

36. Blyth CR: On Simpson's paradox and the sure-thing principle. J Am Stat Assoc 67:364-366, 1972

37. Marcucci G, Maharry K, Wu YZ, et al: IDH1 and IDH2 gene mutations identify novel molecular subsets within de novo cytogenetically normal acute myeloid leukemia: A Cancer and Leukemia Group B study. J Clin Oncol 28:2348-2355, 2010

38. Marková J, Michková $P$, Burèková $K$, et al: Prognostic impact of DNMT3A mutations in patients with intermediate cytogenetic risk profile acute myeloid leukemia. Eur J Haematol 88:128-135, 2012 


\section{AUTHORS' DISCLOSURES OF POTENTIAL CONFLICTS OF INTEREST}

Simpson's Paradox and the Impact of Different DNMT3A Mutations on Outcome in Younger Adults With Acute Myeloid Leukemia

The following represents disclosure information provided by authors of this manuscript. All relationships are considered compensated. Relationships are self-held unless noted. I = Immediate Family Member, Inst = My Institution. Relationships may not relate to the subject matter of this manuscript. For more information about ASCO's conflict of interest policy, please refer to www.asco.org/rwc or jco.ascopubs.org/site/ifc.

\section{Rosemary E. Gale}

No relationship to disclose

\section{Katarina Lamb}

No relationship to disclose

\section{Christopher Allen}

No relationship to disclose

\section{Dima El-Sharkawi}

No relationship to disclose

\section{Cassandra Stowe}

No relationship to disclose

\section{Sarah Jenkinson}

No relationship to disclose

\section{Steven Tinsley}

No relationship to disclose

\section{Glenda Dickson}

No relationship to disclose

Alan K. Burnett

No relationship to disclose

Robert K. Hills

No relationship to disclose

\section{David C. Linch}

Employment: Autolus

Leadership: Autolus

Stock or Other Ownership: Autolus

Travel, Accommodations, Expenses: Gilead Sciences 


\section{Acknowledgment}

We are grateful to the clinical investigators who entered and managed patients in these trials.

\section{Appendix}

\section{Mutation Screening}

Polymerase chain reaction (PCR) products for DNMT3A exons 13 to 23 were amplified from genomic DNA using Optimase Polymerase (Transgenomic, Glasgow, United Kingdom) or Phusion High-Fidelity DNA Polymerase (New England BioLabs, Hitchin, United Kingdom) according to manufacturer's instructions, with 35 cycles of amplification and primers and annealing temperatures as specified (Table A2). Products were denatured, reannealed slowly to allow heteroduplex formation, and then analyzed on a denaturing high-performance liquid chromatography WAVE platform (Transgenomic, Glasgow, United Kingdom) at optimal melting temperatures calculated using Transgenomic's Navigator software (Table A2). Samples with abnormal WAVE chromatograms were sequenced.

For screening of R882H and R882C mutations, PCR products were prepared using BIOTAQ DNA Polymerase (BIOLINE, London, United Kingdom) with 32 cycles of amplification and then digested with restriction enzymes (New England BioLabs) according to manufacturer's instructions. For R882H, the primers were 23F2 and mismatch primer R882H MM (R; Table A2), and the products were digested at $65^{\circ} \mathrm{C}$ for 3 hours with BsaAI. Wild-type products remained uncut ( 172 base pairs [bp]), and mutant products were digested to $143+29 \mathrm{bp}$. For R882C, the primers were $23 \mathrm{~F}$ and $23 \mathrm{R}$, and the products were digested overnight at $37^{\circ} \mathrm{C}$ with AluI. Wild-type products remained uncut ( $318 \mathrm{bp}$ ), and mutant products were digested to $193+125 \mathrm{bp}$.

\section{Quantification of Mutant Level}

For R882H and R882C mutations, the relative mutant level was quantified using pyrosequencing of biotinylated PCR products prepared using GoTaq Hot Start Polymerase (Promega, Southampton, United Kingdom), 50 cycles of amplification, primers Pyro F and biotinylated Pyro R (Table A2), and an annealing temperature of $63^{\circ} \mathrm{C}$. The 184 -bp products were sequenced on a PyroMark MD system (Qiagen, Crawley, United Kingdom) using PyroMark Q96 reagents and protocols (Qiagen) and primer Pyro sequence (Table A2). The pyrosequencing software calculated mutant level as a percentage of the total alleles for a particular mutation.

\section{Definition of Clinical End Points}

Complete remission (CR) was defined as a normocellular bone marrow containing less than $5 \%$ blasts and showing evidence of normal maturation of other marrow elements. CR with incomplete blood count recovery was classified as CR with residual neutropenia or thrombocytopenia. Persistence of myelodysplastic features did not preclude the diagnosis of CR. Remission failures were classified by the clinicians as either a result of induction death (death related to treatment and/or hypoplasia within 30 days of trial entry) or resistant disease (failure to eliminate disease, including partial remission with 5\% to $15 \%$ blasts in the bone marrow). Where the clinician's evaluation was not available, deaths within 30 days of entry were classified as induction deaths, and deaths later than 30 days after entry were classified as resistant disease. Overall survival was defined as the time from random assignment to death. For patients achieving CR, cumulative incidence of relapse was the incidence of relapse after CR with death in CR as a competing risk.

\begin{tabular}{|c|c|c|c|}
\hline Male, \% & 48 & 49 & .7 \\
\hline Median WBC, $\times 10^{9} / \mathrm{L}$ & 26.85 & 11.1 & $<.001$ \\
\hline WHO PS $\geq 2, \%$ & 33 & 36 & .1 \\
\hline CR/CRi, \% & 87 & 84 & .03 \\
\hline OS at 5 years, \% & 39 & 34 & .02 \\
\hline CIR at 5 years, \% & 49 & 53 & .1 \\
\hline
\end{tabular}




\begin{tabular}{|c|c|c|c|c|}
\hline Exon and Primer & Primer Sequence & Size (bp) & $\begin{array}{c}\text { Annealing } \\
\text { Temperature }\left({ }^{\circ} \mathrm{C}\right)\end{array}$ & $\begin{array}{c}\text { WAVE } \\
\text { Temperature }\left({ }^{\circ} \mathrm{C}\right)\end{array}$ \\
\hline \multicolumn{5}{|l|}{13} \\
\hline $\mathrm{F}$ & 5'-GCTGGTCTGGTGCTGGGCTC-3' & 228 & 65 & 63.3 \\
\hline R & 5'-CACAGTCAGCCAGAAGGCCGA-3' & & & \\
\hline \multicolumn{5}{|l|}{14} \\
\hline $\mathrm{F}$ & 5'-TGAGGCCAGGTGTGGAGCCTC-3' & 241 & 67 & 64.1 \\
\hline $\mathrm{R}$ & 5'-TGGGGCCCAGCTAAGGAGACCA-3' & & & \\
\hline \multicolumn{5}{|l|}{15} \\
\hline $\mathrm{F}$ & 5'-TCCATTCCAGGTAGCACACCTTG-3' & 338 & 63 & $63.4,64.0$ \\
\hline R & 5'-ACCCTGCGCACAGCTCAGGC-3' & & & \\
\hline \multicolumn{5}{|l|}{16} \\
\hline $\mathrm{F}$ & 5'-GACACCGCTGGGCCTGCATC-3' & 221 & 66 & 62.6 \\
\hline R & 5'-ACCATCATTTCGTTTTGCCAGAGTTGC-3' & & & \\
\hline \multicolumn{5}{|l|}{17} \\
\hline $\mathrm{F}$ & 5'-TGCCGAGACCAGGGTGCCAG-3' & 269 & 66 & 64.1 \\
\hline $\mathrm{R}$ & 5'-CTCCAGGTGCTGAGTGTGCAG-3' & & & \\
\hline \multicolumn{5}{|l|}{18} \\
\hline $\mathrm{F}$ & 5'-CTGGGTCTCCTCTCTTTCGTG-3' & 252 & 63 & 62.6 \\
\hline $\mathrm{R}$ & 5'-GCACCAGCTGAGAAGGTGGAG-3' & & & \\
\hline \multicolumn{5}{|l|}{19} \\
\hline $\mathrm{F}$ & 5'-AGCCACACCACTGTCCTATGC-3' & 304 & 63 & $61.5,62.7$ \\
\hline $\mathrm{R}$ & 5'-TCCCCAGCTCCACAATGCAGAT-3' & & & \\
\hline \multicolumn{5}{|l|}{20} \\
\hline $\mathrm{F}$ & 5'-CTTTAAGGCTCGACCCCAGCA-3' & 241 & 64 & 62.2 \\
\hline $\mathrm{R}$ & 5'-GCTTCCCCACTATGGGTCATC-3' & & & \\
\hline \multicolumn{5}{|l|}{21} \\
\hline $\mathrm{F}$ & 5'-GAGGGAGGGGAGTCGTGCA-3' & 267 & 63 & 62.4 \\
\hline $\mathrm{R}$ & 5'-GCATTCTCCACACTAGCTGGAGA-3' & & & \\
\hline \multicolumn{5}{|l|}{22} \\
\hline $\mathrm{F}$ & 5'-GAGTACCTGGCATATTTGGTAGAC-3' & 297 & 64 & 59.0 \\
\hline $\mathrm{R}$ & 5'-CAAGTCAGGTGGGAAAGGCAG-3' & & & \\
\hline \multicolumn{5}{|l|}{23} \\
\hline $\mathrm{F}$ & 5'-CCTGCTGTGTGGTTAGACGGCT-3' & 318 & 64 & $60.8,63.6$ \\
\hline $\mathrm{R}$ & 5'-СTCTCСАTССTCATGTTCTTGGTG-3' & & & \\
\hline F2 & 5'-CTGGTCCTCCGGGTCCTGC-3' & 172 & 64 & NA \\
\hline R882H MM (R) & 5'-GACCGGCCCAGCAGTCTCTGCCTCGCCACG-3'* & & & \\
\hline Pyro F & 5'-TGTGTGGTTAGACGGCTTCC-3' & 184 & 63 & NA \\
\hline Pyro R (biotin) & 5'-GAAGAGGTGGCGGATGACT-3' & & & \\
\hline Pyro sequence & 5'-TGACGTCTCCAACATGA-3' & & & \\
\hline
\end{tabular}




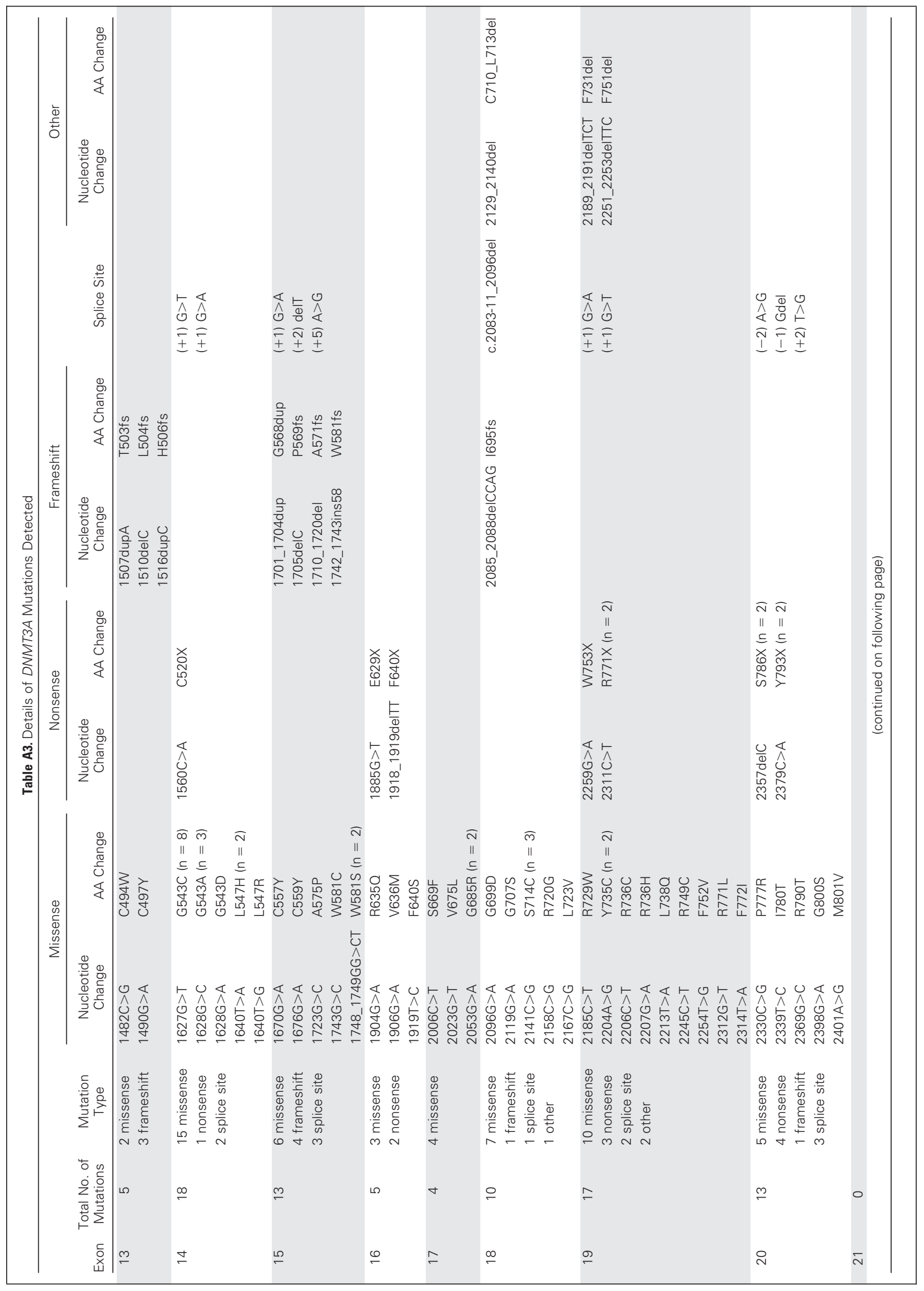




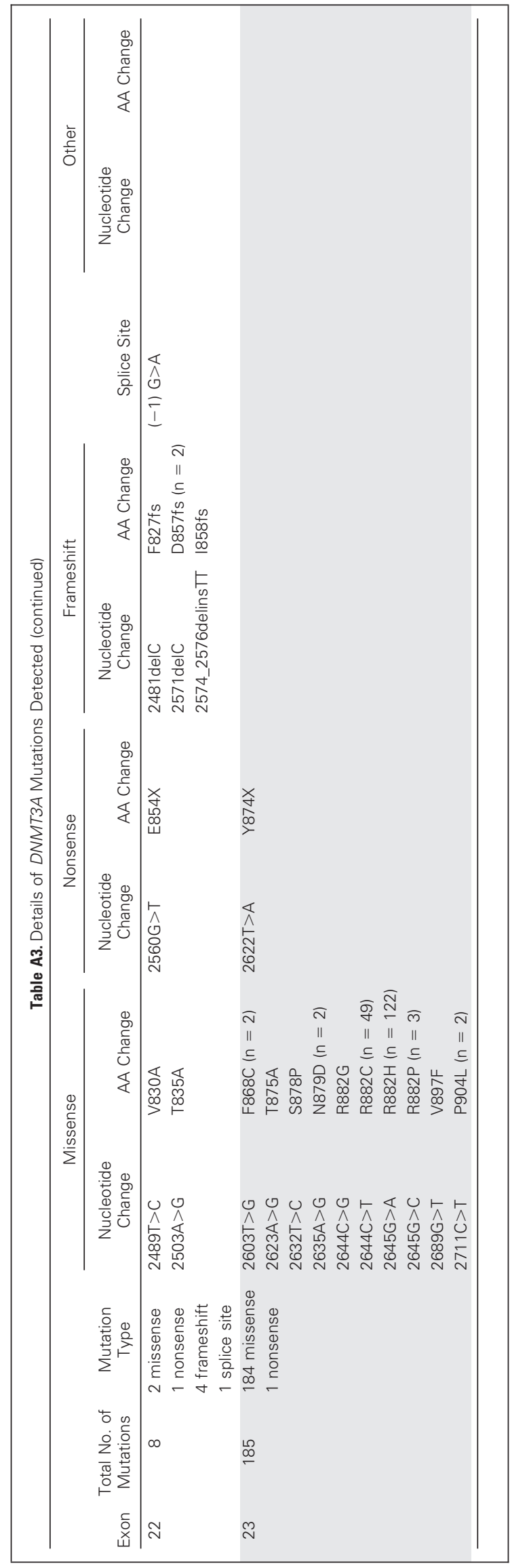


Table A4. Multivariable Analysis of Outcome in the Total Cohort

\begin{tabular}{|c|c|c|c|c|c|c|c|}
\hline \multirow[b]{3}{*}{ Outcome } & \multirow{2}{*}{\multicolumn{2}{|c|}{ MUT $v$ WT }} & \multicolumn{2}{|r|}{$P$} & & & \multirow{3}{*}{$\begin{array}{c}\text { R882 } v \text { Non-R882 } \\
\text { Missense } v \text { Truncation, } P\end{array}$} \\
\hline & & & \multirow{2}{*}{$\begin{array}{c}\text { WT } v \text { R882 } v \\
\text { Others }\end{array}$} & \multirow{2}{*}{$\begin{array}{l}\text { WT } v \text { R882 } v \text { Non-R882 } \\
\text { Missense } v \text { Truncation }\end{array}$} & \multicolumn{2}{|c|}{ R882 v Others } & \\
\hline & $\mathrm{HR}(95 \% \mathrm{Cl})$ & $P$ & & & HR $(95 \% \mathrm{Cl})$ & $P$ & \\
\hline $\mathrm{CR} / \mathrm{CRi}$ & $0.74(0.43$ to 1.28$)$ & .3 & .2 & .4 & 1.97 (0.67 to 5.77$)$ & .2 & .5 \\
\hline OS & 1.19 (0.98 to 1.45$)$ & .1 & .1 & .1 & 1.19 (0.87 to 1.64$)$ & .3 & .2 \\
\hline $\mathrm{CIR}$ & 1.27 (1.01 to 1.61$)$ & .04 & .1 & .05 & 1.25 (0.85 to 1.85$)$ & .3 & .2 \\
\hline
\end{tabular}

NOTE: Results were adjusted for age, WHO performance status, log(WBC), secondary disease, FLT3 ${ }^{\mid T D}$, and NPM1 genotype.

Abbreviations: CIR, cumulative incidence of relapse; CR, complete remission; CRi, complete remission with incomplete hematologic recovery; HR, hazard ratio; MUT, mutant; OS, overall survival; WT, wild type.

\begin{tabular}{|c|c|c|}
\hline Variable & HR $(95 \% \mathrm{Cl})$ & $P$ \\
\hline \multicolumn{3}{|l|}{ CR } \\
\hline NPM1 ${ }^{\mathrm{MUT}}$ & 0.21 (0.13 to 0.34$)$ & $<.001$ \\
\hline Age & 1.05 (1.03 to 1.07 ) & $<.001$ \\
\hline WHO PS & 1.52 (1.26 to 1.83$)$ & $<.001$ \\
\hline $\log (\mathrm{WBC})$ & 2.19 (1.52 to 3.14$)$ & $<.001$ \\
\hline Secondary disease & 2.51 (1.31 to 4.81 ) & .005 \\
\hline \multicolumn{3}{|l|}{$\mathrm{CIR}$} \\
\hline$F L T 3^{\mid T D}$ & 2.14 (1.73 to 2.65$)$ & $<.001$ \\
\hline$N P M 1^{M U T}$ & 0.39 (0.31 to 0.49 ) & $<.001$ \\
\hline Age & 1.01 (1.00 to 1.02$)$ & .003 \\
\hline CEBPA ${ }^{\mathrm{DM}}$ & 0.57 (0.36 to 0.89 ) & .01 \\
\hline DNMT3A $A^{\mathrm{MUT}}$ & 1.27 (1.00 to 1.61$)$ & .05 \\
\hline \multicolumn{3}{|l|}{ OS } \\
\hline NPM1 $1 \mathrm{MUT}$ & $0.42(0.35$ to 0.50$)$ & $<.001$ \\
\hline Age & 1.02 (1.02 to 1.03 ) & $<.001$ \\
\hline FLT3'TD & 1.55 (1.30 to 1.85$)$ & $<.001$ \\
\hline WHO PS & $1.13(1.05$ to 1.22$)$ & $<.001$ \\
\hline CEBPA ${ }^{\mathrm{DM}}$ & 0.43 (0.28 to 0.65$)$ & $<.001$ \\
\hline $\log (W B C)$ & 1.26 (1.10 to 1.45$)$ & .001 \\
\hline \multicolumn{3}{|c|}{$\begin{array}{l}\text { NOTE. Models were fitted using forward selection, with variables added to the model if they had a } P<.05 \text {, derived using the deviance statistic. Variables entered } \\
\text { were age, WHO PS, log(WBC), secondary disease, } F L T 3^{T T}, F L T 3^{T K D}, N P M 1, C E B P A^{D M}, I D H 1, I D H 2 \text {, and DNMT3A genotype. DNMT3A was entered as mutant/not, } \\
\text { WT } v \text { R882 } v \text { other, WT } v \text { R882, and missense } v \text { truncation. } \\
\text { Abbreviations: CIR, cumulative incidence of relapse; CR, complete remission; DM, double mutant; HR, hazard ratio; ITD, internal tandem duplication; MUT, mutant; } \\
\text { OS, overall survival; PS, performance status; TKD, tyrosine kinase domain; WT, wild type. }\end{array}$} \\
\hline
\end{tabular}

Table A6. Contingency Table for OS in the Different Genotype Groups

\begin{tabular}{|c|c|c|c|c|c|}
\hline \multirow[b]{2}{*}{ Genotype } & \multicolumn{2}{|c|}{ DNMT3AWT } & \multicolumn{2}{|c|}{ DNMT3AMUT } & \multirow[b]{2}{*}{$\begin{array}{l}\text { Total No. of } \\
\text { Patients }\end{array}$} \\
\hline & $\begin{array}{l}\text { No. of Patients ( } \% \text { of } \\
\text { DNMT3A subgroup) }\end{array}$ & $\begin{array}{c}\text { Observed } 10-\text { Year OS } \\
\text { Rate }(\%)\end{array}$ & $\begin{array}{l}\text { No. of Patients (\% of } \\
\text { DNMT3A subgroup) }\end{array}$ & $\begin{array}{c}\text { Observed } 10-\text { Year OS } \\
\text { Rate }(\%)\end{array}$ & \\
\hline NPM1WT & $401(62)$ & 25 & $54(20)$ & 15 & 455 \\
\hline$N P M 1^{\mathrm{MUT}}$ & $241(38)$ & 50 & $218(80)$ & 38 & 459 \\
\hline Total & 642 & & 272 & & 914 \\
\hline
\end{tabular}

NOTE. The survival for each DNMT3A group (DNMT3A ${ }^{\mathrm{WT}}$ and DNMT3A ${ }^{\mathrm{MUT}}$ ) is obtained by adding together the proportion of NPM $1^{\mathrm{WT}}$ patients multiplied by their survival and the proportion of NPM1 ${ }^{\mathrm{MUT}}$ patients multiplied by their survival. For DNMT3A ${ }^{\mathrm{WT}}, \mathrm{OS}=(0.62 \times 25 \%)+(0.38 \times 50 \%)=34 \%$. For DNMT3A $A^{\mathrm{MUT}}, \mathrm{OS}=$ $(0.20 \times 15 \%)+(0.80 \times 38 \%)=33 \%$.

Abbreviations: MUT, mutant; OS, overall survival; WT, wild type. 


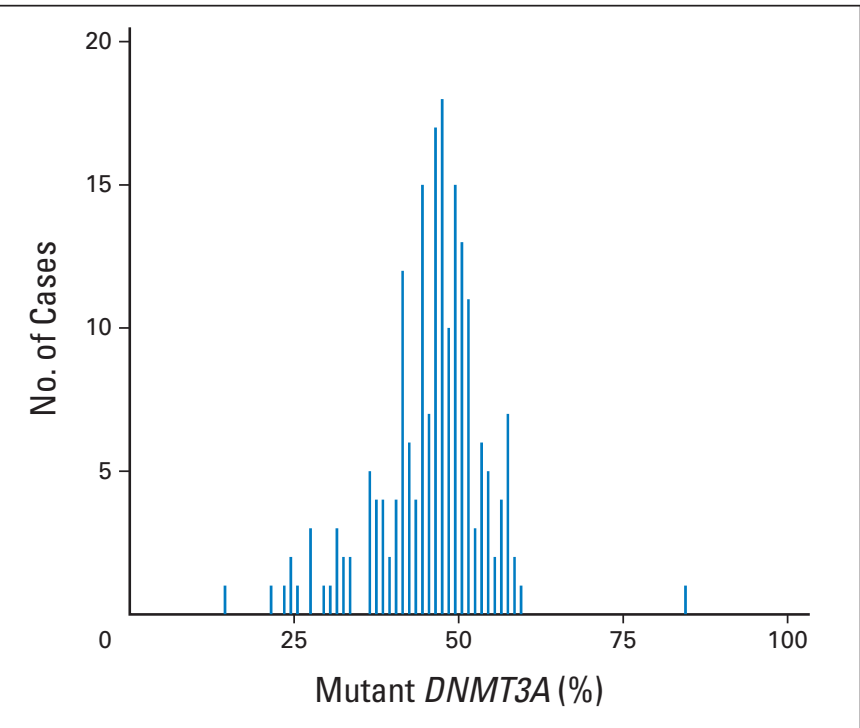

Fig A1. Distribution of relative mutant level quantified in 172 patients with DNMT3A R882H or R882C mutations. 